\title{
Wideband Integrated Optical Delay Line Based on a Continuously Tunable Mach-Zehnder Interferometer
}

\author{
Daniele Melati, Abi Waqas, Zarlish Mushtaq, and Andrea Melloni
}

\begin{abstract}
An integrated optical delay line is presented and experimentally demonstrated with a true-time delay continuously tuned up to 125 ps. The proposed device is based on a MachZehnder interferometer with tuneable couplers, can be ideally operated with a single control signal and achieves a bandwidth delay product consistently larger than ring-based delay lines. The device is successfully used in a transmission system to control the delay of a $10 \mathrm{Gbit} / \mathrm{s}$ data stream.
\end{abstract}

Index Terms-Optical delay lines, true-time delay, microwave photonics, optical filters, MachZehnder interferometer, integrated photonics, indium phosphide waveguides.

\section{INTRODUCTION}

$\mathbf{O}$ PTICAL true-time delay lines allow to arbitrary control the propagation delay of an optical signal and can be exploited for a variety of applications. Typical examples include synchronization and buffering of optical signals [1][3]; optical signal processing and microwave photonic systems [4]; optical coherence tomography [5]; optical beam forming networks for phased-array antennas [6], [7], where the use of optical true-time delay lines increases the bandwidth of the antenna and avoids beam squinting [4].

Photonic integration technologies are particularly advantageous for the realization of (tunable) optical delay lines since allow to reduce costs and sizes of the devices [8] and ease the integration with other optical functions such as filtering [9], modulation and light generation. Many different schemes have been proposed for the realization of integrated optical delay lines, relying on different types of optical filters [10], [11]. The first broad class comprises all the devices that exploits resonant enhancement. This includes both the exploitation of material resonances, inducing large dispersion and thus small group velocities, and "circuit" resonance (i.e. infinite impulse-response filters), forcing light to recirculate and hence increasing the group delay. Among others, ring resonators are very common devices to realize a circuit resonator and tune group delay up to several hundreds of picoseconds [12]-[14], possibly exploiting multiple resonators either with coupled resonator optical waveguide designs (CROW) [15] or sidecoupled integrated spaced sequence of resonators (SCISSORs) [16]. Delay lines based on photonic crystals can be included in this class as well [14]. Although resonant delay lines make possible to obtain large delays with very compact devices,

D. Melati, A. Waqas, and A. Melloni are with the Dipartimento di Elettronica, Informazione e Bioningegneria, Politecnico di Milano, via Ponzio 34/5, 20133 Milano, Italy. A. Waqas and Z. Mushtaq are with the Department of Telecommunication Engineering, Mehran University of Engineering and Technology, Jamshoro, 76062 Sindh, Pakistan. e-mail: daniele.melati@ polimi.it

Manuscript received XXXXX XX, XXXX; revised XXXXXX XX, XXXX. this generally comes at the expense of large losses and narrow bandwidth [10]. Coupled resonators can increase the operational bandwidth but generally require complex tuning strategies.

The second class of delay lines includes structures that do not employ resonant enhancement. Group delay tunability is in the order of the size of the device and is generally smaller compared to resonant delay lines. On the other hand, nonresonant structures guarantee a much larger bandwidth and a finer control of the tuning. The most common integrated devices comprised in this class are switched delay lines where path with different physical lengths are selected by a series of switches thus changing the total propagation length and delay [17]. Their use has been proposed also in combination with ring resonators [18]. The drawback of this solution is the need of a large number of switches with a high on-off extinction ratio to guarantee the proper functionality. Further, continuous tune of the delay is not possible since the control is necessarily done by discrete steps. A non-resonant delay line implementing a finite impulse-response filter (Mach-Zehnder) has been proposed and realized with fibre-based components exploiting either dispersive media [19] or a polarizationdomain Mach-Zehnder interferometer [20]. Because of the use of a single tunable coupler, the amplitude of the light travelling through this device necessarily depends on the applied delay, also in the ideal lossless case [21]. Ring resonators operated far from resonance wavelengths could be included in the class of non-resonant structures [9].

In this work we propose an architecture to realize a nonresonant optical delay line based on a Mach-Zehnder interferometer equipped with two tunable couplers. For a given delay tuning range, this solution achieves a true-time delay operation on a larger bandwidth (calculated on the group delay spectra) and induces a smaller dispersion compared to ringbased delay lines, even used at off-resonance wavelengths.

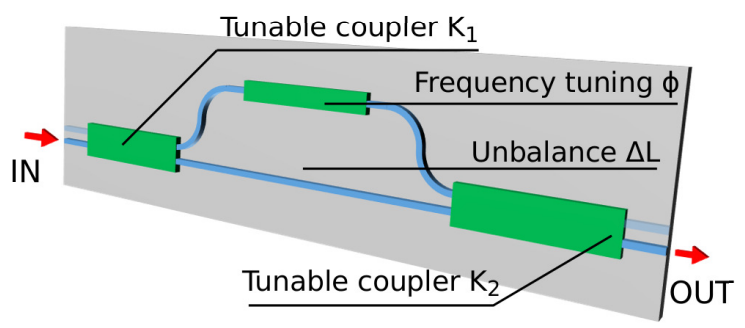

Fig. 1. Sketch of the proposed optical delay line based on a Mach-Zehnder interferometer with two tunable couplers. 
Group delay is tuned in a continuous manner by using a single control signal to vary the coupling ratio of both couplers, thus simplifying the operation. At the operative wavelength, the power transfer function of the device does not depend on the tuning of the group delay (for the ideal lossless case), preventing any modulation of the amplitude of the optical signal due to the reconfiguration of the delay line. The device is realized on an InP-based photonic integration platform and experimentally demonstrated with a group delay tunability up to $125 \mathrm{ps}$. Although the proposed delay line does not depend on any particular integration technology, the use of a platform based on InP may ease the integration of the device with highperformance active components, such as fast tunable couplers or photodiodes.

The paper is organized as follow. Section II presents the operational principle and the design of the proposed delay line. Performance is evaluated in terms of delay tuning, bandwidth and induced dispersion and compared with ring-based delay lines. Section III describes the realization of the circuit and the experimental characterization of the tunable couplers and the delay line, reporting both power transmission spectra and group delay spectra. Polarization dependence is carefully investigated and the continuous control of the delay of a 10 Gbit/s OOK NRZ signal is demonstrated. Lastly, conclusions and outlook are presented.

\section{Mach-Zehnder-BASed Optical Delay Line}

This section describes the design and operation of the proposed optical delay line based on a continuously tunable Mach-Zehnder interferometer. The same device could be realized also exploiting a Michelson scheme, using the same control strategy while implementing only one tunable coupler and obtaining exactly the same behaviour. This configuration is not discussed in this work.

\section{A. Design and Functionality}

The proposed integrated optical delay line is schematically shown in Fig. 1. The circuit implements a Mach-Zhender interferometer with two tunable couplers whose coupling ratios $\mathrm{K}_{1}$ and $\mathrm{K}_{2}$ can be varied from 0 to 1 . Tunable couplers can be realized for example with two balanced Mach-Zhender couplers equipped with phase shifters, as experimentally demonstrated in Sec. III-A. The interferometer has an unbalancing $\Delta \mathrm{L}$ between the two branches and a phase shifter integrated on one of the arms allows to tune the operative frequency of the device to match that of the incoming signal, as described below. The time unbalance between the two arms of the interferometer is defined as

$$
T=\frac{n_{g} \Delta L}{c}=\frac{1}{F S R}
$$

where $\mathrm{n}_{g}$ is the group index of the waveguide, $\mathrm{c}$ is the speed of light and FSR is the Free Spectral Range.

In the most general case this interferometric structure has two input ports and two output ports but in this work it is used as a single-input single-output circuit in bar state [see Fig. 1] to control in a continuous mode the delay of the light travelling through the device. To this purpose, the two coupling ratios are chosen such that $\mathrm{K}_{1}=\mathrm{K}_{2}=\mathrm{K}$, thus actually controlling the couplers with a single control signal. The same behaviour described below could be obtained also using the interferometer in cross state but choosing the coupling coefficients $\mathrm{K}_{1}=1-\mathrm{K}_{2}$. Being $\mathrm{H}$ the transfer function of the interferometer in bar state and $\tau=\partial(\angle \mathrm{H}) / \partial(2 \pi \mathrm{f})$ the corresponding group delay (with $\angle \mathrm{H}$ the phase of $\mathrm{H}$ ), the normalized group delay $\tilde{\tau}=\left(\tau-\tau_{0}\right) / T$ can be written as

$$
\tilde{\tau}=\frac{K(K-\cos \Delta \varphi+K \cos \Delta \varphi)}{2 K^{2} \cos \Delta \varphi-2 K \cos \Delta \varphi-2 K+2 K^{2}+1}
$$

where $\mathrm{T}$ is defined by Eq. (1), $\tau_{0}$ is the minimum delay, associated to the shortest branch of the interferometer, $\Delta \varphi(f)=2 \pi f \Delta L / c+\phi$ is the phase difference between the light propagating in the two branches of the device, $f$ is the light frequency, and $\phi$ is a constant phase shift applied through the phase shifter integrated in the interferometer [see Fig. 1]. Changing $\phi$ the transfer function of the delay line is tuned in the spectral domain. The minimum group delay $\tau=\tau_{0}$ hence corresponds to $\tilde{\tau}=0$ while $\tilde{\tau}=1$ corresponds to $\tau=\tau_{0}$ $+\mathrm{T}$, the maximum group delay obtained travelling through the longest branch of the interferometer. The simulation of the intensity transmission $|\mathrm{H}|^{2}$ of the circuit is reported in Fig. 2(a) while Fig. 2(b) shows the normalized group delay $\tilde{\tau}$. Both are reported as function of the normalized frequency $\tilde{f}=\left(f-f_{o}\right) / F S R$ for different values of $\mathrm{K}$ from 0 to 1 and neglecting propagation losses. $f_{o}$ is a frequency such that $\Delta \varphi\left(f_{o}\right)=(2 N+1) \pi$, with $\mathrm{N}$ integer. According to the scheme shown in Fig. 1, when $\mathrm{K}=0$ the entire input power is coupled to the shortest branch of the Mach-Zehnder and then to the output port. The power transfer function and the group delay are hence spectrally flat, $|\mathrm{H}|^{2}=1$ and $\tilde{\tau}=0$ [yellow lines]. Increasing $\mathrm{K}$, input power is subdivided between the two arms of the interferometer and recombined at the output
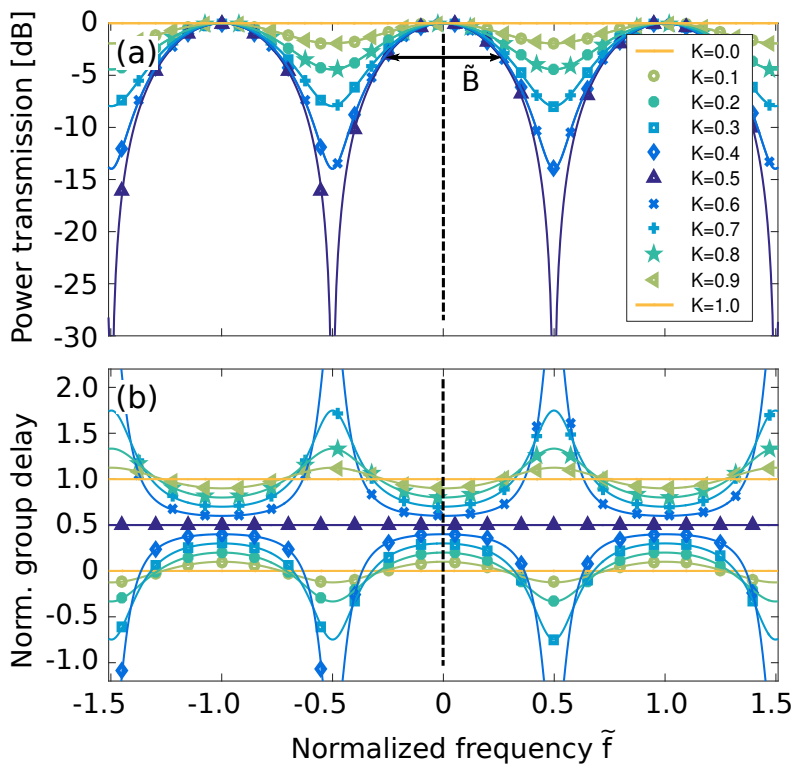

Fig. 2. Simulation of (a) the power transfer function $|\mathrm{H}|^{2}$ and (b) the normalized group delay $\tilde{\tau}=\left(\tau-\tau_{0}\right) / T$ of the delay line as function of the normalized frequency $\tilde{f}=\left(f-f_{o}\right) / F S R$. Dashed lines mark the operative frequency of the device while $\tilde{B}$ is the normalized 3-dB bandwidth. 



Fig. 3. (a) Normalized group delay and (b) bandwidth-delay product calculated at $\tilde{f}=0$ as function of the coupling coefficient K. (c) Group delay dispersion as funcition of the normalized frequency for the value of $\mathrm{K}$ that generates the minimum bandwidth. Data are reported for the Mach-Zehnder delay line [blue solid curve], an all-pass ring resonator used out of resonance [red dashed line] and the same ring used at the resonance frequency [yellow dot-dashed line].

with the same coefficient. For this reason, maxima and minima appear on the power transfer function and group delay of the device [green to blue marks]. The deepest notches in the power spectrum are reached with $\mathrm{K}=0.5$, that causes the group delay to be again spectrally flat with $\tilde{\tau}=0.5$. With $\mathrm{K}=1$ light travels only on the longest branch of the Mach-Zehnder and leaves again the interferometer from the bar port. In this case $|\mathrm{H}|^{2}$ $=1$ and $\tilde{\tau}=1$ [yellow lines]. The same behaviour can be obtained also using the other bar state (entering and leaving the device from the two upper ports in Fig.1), with the only difference that $\tilde{\tau}$ changes from 1 to 0 when $\mathrm{K}$ increases from 0 to 1 .

Interestingly, at the frequency $f_{o}$ (corresponding to $\tilde{f}=0$, represented in Fig. 2 with dashed lines) the intensity transfer function of the delay line is constant and does not depend on $\mathrm{K}$. We can hence define $f_{o}$ as the operative frequency of the device. At this operative frequency, the group delay is linearly dependent on $\mathrm{K}$, as can be easily demonstrated through Eq. (2):

$$
\left|H\left(f_{o}\right)\right|^{2}=1, \quad \tilde{\tau}\left(f_{o}\right)=K .
$$

For this reason, if the bandwidth of the signal travelling through the delay line is sufficiently small, its delay can be changed in a continuous manner between $\tau_{0}$ and $\tau_{0}+\mathrm{T}$ without introducing any extra loss or filtering effect due to variations of the coupling ratio $\mathrm{K}$ hence leaving the signal undistorted. For this structure the normalized 3-dB bandwidth around $f_{o}$ as indicated in Fig. 2(a) can be calculated as

$$
\tilde{B}\left(f_{o}\right)=\frac{B\left(f_{o}\right)}{F S R}=\frac{1}{\pi} \arccos \left[\frac{(K-0.5)^{2}}{K(1-K)}\right] .
$$

As expected, the minimum bandwidth is reached at $\mathrm{K}=0.5$, where $\tilde{B}\left(f_{o}\right)=0.5$ (corresponding to $B\left(f_{o}\right)=F S R / 2$ ) and $\tilde{\tau}\left(f_{o}\right)=0.5\left(\tau\left(f_{o}\right)=T / 2\right)$.

\section{B. Performance and Comparison with Ring Resonators}

The performance of the proposed delay line is evaluated here with a general approach in terms of maximum delay tunability, bandwidth and induced dispersion on the signal with respect to the widely exploited delay lines based on optical all-pass ring resonators. The Mach-Zehnder-based delay line exhibits a larger bandwidth-delay product (i.e. a larger bandwidth for a given delay) and a smaller dispersion compared to ring resonators. The ring delay line considered for the comparison has a single ring resonator equipped with a tunable coupler that allows to control the amount of optical power $\mathrm{K}$ coupled in and out of the resonant cavity. Also for this structure the normalized group delay as function of frequency and coupling coefficient $\mathrm{K}$ can be analytically calculated [22]. The comparison is done through normalized quantities and hence the actual unbalance of the Mach-Zehnder and length of the ring do not play a role and results can be applied to delay lines of any length. In both cases losses are neglected. Since in the ideal case taken into account here the 3-dB bandwidth cannot be defined for an all-pass filter, the bandwidth is measured directly on the spectrum of the group delay response for both the ring and the Mach-Zhender. In particular, the normalized bandwidth is defined as the difference between the two normalized frequencies around $\tilde{f}=0$ (the operative frequency) where the delay $\tilde{\tau}$ drops by $5 \%$.

The dependence of the normalized group delay $\tilde{\tau}$ on the coupling coefficient $\mathrm{K}$ at the operative frequency of the delay lines is shown in Fig. 3(a). Blue line refers to the MachZhender interferometer. As already discussed, in this case $\tilde{\tau}=$ $\mathrm{K}$ meaning that the group delay changes linearly with $\mathrm{K}$ from $\tau_{0}$ to $\tau_{0}+\mathrm{T}$. For the ring, two different operative frequencies are considered. The first one is the off-resonance frequency, such that the round-trip phase delay of the ring is equal to $(2 N+1) \pi$ [red dashed line]. At this frequency the group delay of the ring has its minimum and is not affected by the resonance. When $\mathrm{K}=0$ light does not couple in the ring and just travels in the bus, experiencing the minimum delay. When $\mathrm{K}=1$ all the light is coupled in the ring and back in the bus after a single round-trip, experiencing the maximum delay. The behaviour is hence very similar to the Mach-Zehnder structure and $\tilde{\tau}$ grows from 0 (with $\mathrm{K}=0$ ) to 1 (with $\mathrm{K}=$ 1). The difference is that for the ring the dependence on $\mathrm{K}$ is highly non-linear. The second possible operative condition for the ring is the resonant frequency, such that the roundtrip phase delay is equal to $2 N \pi$ [yellow dot-dashed line]. On resonance, the group delay of the ring has its minimum $\tilde{\tau}=1$ when $\mathrm{K}=1$ and steeply increases with decreasing K. For the Mach-Zhender, the minimum normalized bandwidth (defined 


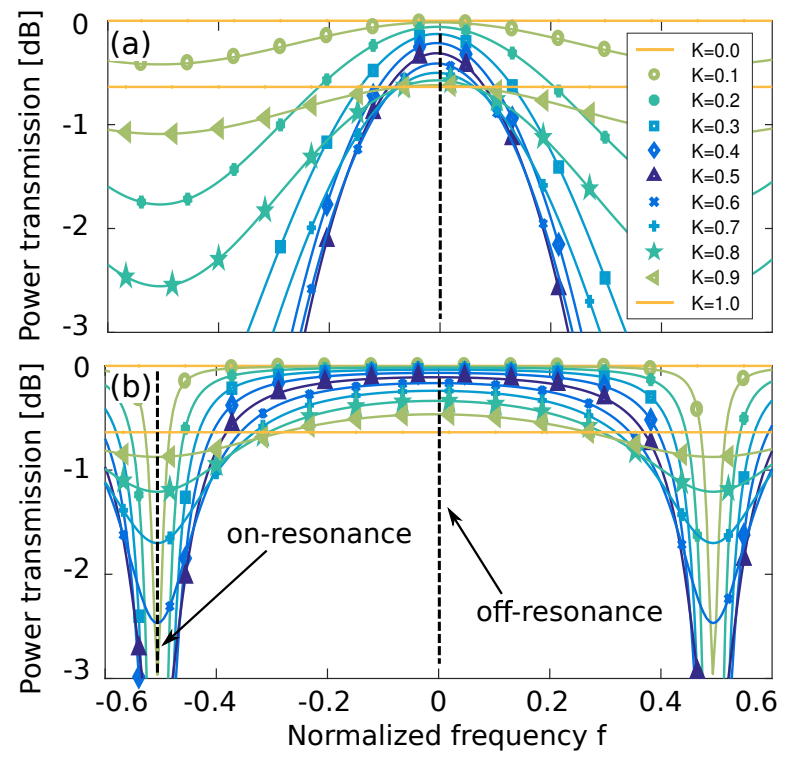

Fig. 4. Simulation of the power transfer function $|\mathrm{H}|^{2}$ with propagation losses for (a) the Mach-Zehnder delay line and (b) the all-pass ring resonator. Possible operative frequencies are marked with dashed lines (both on-resonance and off-resonance conditions are highlighted for the ring).

on the group delay) is about 0.31 , reached for $\mathrm{K}$ equal to 0.25 and 0.75 . For the ring resonator used out of resonance, minimum normalized bandwidth of 0.22 is reached at $\mathrm{K} \simeq$ 0.9. On resonance, the ring has an almost null normalized bandwidth with $\mathrm{K} \simeq 0$ (ideal case without losses). Onresonance operation hence guarantees the largest continuous delay tunability but at the expense of narrow bandwidths [10].

In order to better compare the performance of the considered delay lines in terms of delay and bandwidth (calculated on the group delay spectra), Fig. 3(b) shows the value of the bandwidth - delay product as function of the coupling coefficient $\mathrm{K}$ for the three cases. As can be seen, a ringresonator-based delay line has a product value that is almost identical when used in resonance or off resonance [red dashed and yellow dot-dashed line, respectively]. The bandwidth delay product slowly grows with $\mathrm{K}$ up to about 0.15 (excluding values of $\mathrm{K}$ either close to 0 or close to 1 ). The larger group delay of the ring when used at the resonance frequency is compensated by a narrow bandwidth while off resonance the opposite condition apply: a lower group delay is guaranteed on a much larger bandwidth. The delay line based on a MachZehnder interferometer has a bandwidth - delay product that is always larger than that of the ring and grows with $\mathrm{K}$. The product goes to infinite for $\mathrm{K}=0, \mathrm{~K}=0.5$ and $\mathrm{K}=1$ because for these values the bandwidth (defined on the group delay) is infinite. For a given value of the group delay, the bandwidth of the Mach-Zehnder delay line is hence always larger than the bandwidth of a ring-resonator-based delay line, even when used out of resonance.

Figure 3(c) shows the value of the normalized group delay dispersion $\partial \tilde{\tau} / \partial(2 \pi \tilde{f})$ as function of $\tilde{f}$. Dispersion is calculated in the three cases at the value of $K$ that gives the minimum value of the bandwidth $(K=0.75$ for the MachZehnder, about $\mathrm{K}=0.9$ for the ring out of resonance and

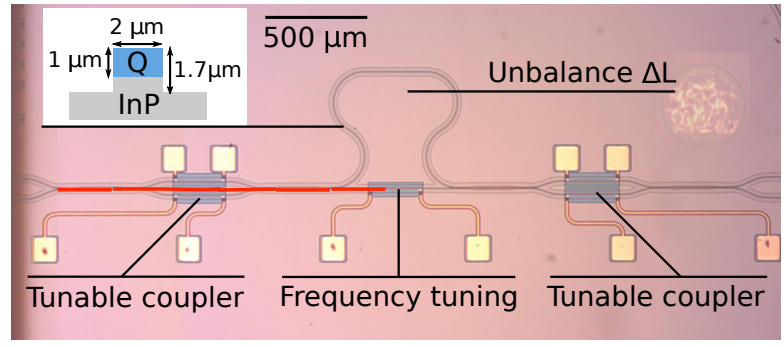

Fig. 5. Photograph of the realized Mach-Zehnder-based tunable delay line.

$\mathrm{K}$ close to zero for the ring used at the frequency of the resonance). The value of the bandwidth (defined on the group delay spectra) is reported for the Mach-Zehnder and offresonance ring. For all the three cases the dispersion is zero at the operative frequency of the delay lines $(\tilde{f}=0)$. As expected, the ring resonator used on resonance [yellow dotdashed line] exhibits a very high dispersive behaviour around $\tilde{f}=0$. On the contrary, the Mach-Zehnder [blue solid line] and the off-resonance ring [red dashed line] have a much smoother and similar dependence of the dispersion on the frequency. The maximum dispersion for the Mach-Zehnder is reached around $\tilde{f}=0.4$ and it is equal to about 0.5 . The ring has a maximum dispersion that is almost twice that of the MachZehnder at the same frequency. In general, the dispersion of the Mach-Zehnder is always lower than the dispersion of the off-resonance ring.

Lastly, the effect of propagation losses on the intensity transmission $|\mathrm{H}|^{2}$ of both the Mach-Zehnder and the all-pass ring is shown in Fig. 4 for several values of the coupling coefficient $\mathrm{K}$ between 0 and 1 . As an example, during the simulations a differential attenuation between the two arms of the Mach-Zhender of about $0.7 \mathrm{~dB}$ was considered. Likewise, the considered round-trip loss of the ring is about $0.7 \mathrm{~dB}$. The operative frequencies for the two devices are marked with dashed lines. Light travelling in the all-pass ring resonator experiences a considerably high attenuation at the resonant frequency, in particular when the coupling coefficient $\mathrm{K}$ is close to the critical coupling condition. This actually limits the maximum delay that can be practically achieved with this structure. On the contrary, when light is at the off-resonance frequency of the ring, the differential attenuation between the two conditions $\mathrm{K}=0$ and $\mathrm{K}=1$ is only due to the propagation losses of a single round-trip in the ring and it is hence much smaller. The same is true for the Mach-Zehnderbased delay line, where the differential attenuation is related to the difference between the propagation losses for the two arms (and hence to the unbalance). In this case the the insertion loss grows linearly with $\mathrm{K}$. The dependence of the group delay on the coupling coefficient $\mathrm{K}$ is similar to the lossless case shown in Fig. 3(a) except for the critical coupling condition of the ring that changes for different values of round-trip loss.

\section{Circuit FAbrication And Experimental Results}

Figure 5 shows the photograph of the tunable optical delay line fabricated through a JePPIX Multi-Project Wafer Run [23] on an InP-based technological platform [24]. As shown in the 

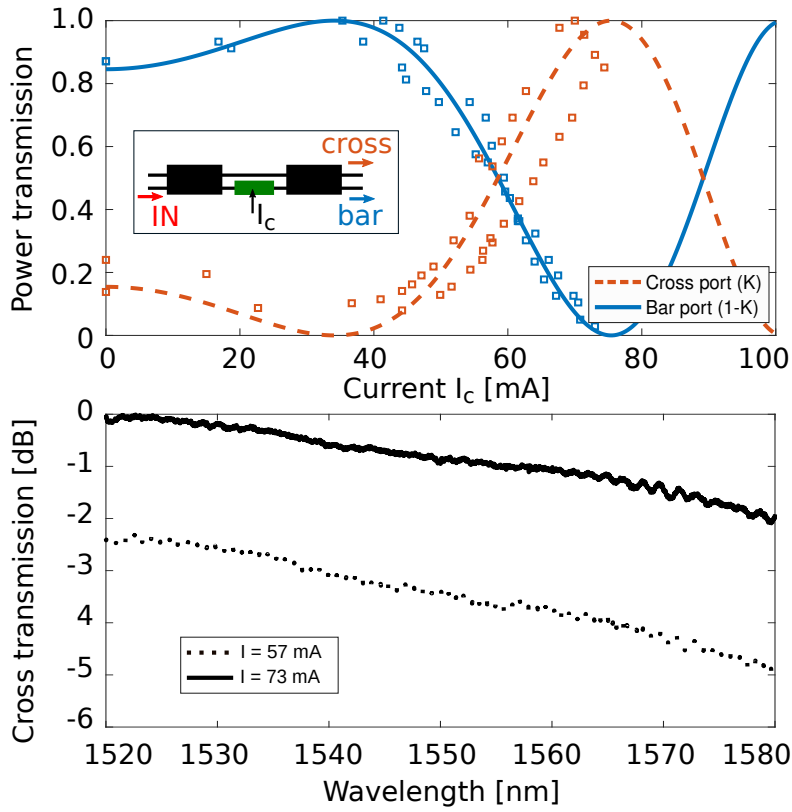

Fig. 6. (a) Power transmission at the bar and cross ports of the tunable coupler as function of the driving current. The schematic of the tunable coupler is shown in the inset. (b) Power spectrum of the cross port of the coupler for two different values of the fed current.

inset, the exploited waveguide is rib-shaped with $1 \mu \mathrm{m}$-thick InGaAsP core $(\mathrm{Q})$ on top of the InP substrate, etch depth of $1.7 \mu \mathrm{m}$ and no top cladding. Waveguide width is $2 \mu \mathrm{m}$ and chip facets are covered with an anti-reflective coating guaranteeing a reflection coefficient smaller than $0.1 \%$ at $\lambda$ $=1550 \mathrm{~nm}$ [8]. Expected propagation losses of the exploited waveguides are about $2 \mathrm{~dB} / \mathrm{cm}$ [24]. The minimum radius of the designed bent waveguides is $150 \mu \mathrm{m}$. The tunable couplers are realized through two additional balanced $2 \times 2$ Mach-Zehnder interferometers with 3-dB MMI couplers and 250- $\mu \mathrm{m}$-long thermo-optic phase shifters, as shown also in the inset of Fig. 6(a). The same phase shifter is integrated also on one of the arm of the unbalanced Mach-Zehnder to tune the operative frequency of the delay line. Two delay lines were realized with an unbalancing $\Delta \mathrm{L}$ of $10.5 \mathrm{~mm}$ (long delay line) and $1.275 \mathrm{~mm}$ (short delay line) [Fig. 5], respectively. In both cases the total length of the device is comprised between 3.5 $\mathrm{mm}$ and $4 \mathrm{~mm}$. As discussed in section II-A, the device has two input ports and two output ports but for the purposes of this work it is used as a $1 \times 1$ device in bar state. The unused output port (cross state) could be exploited to provide a feedback for the tuning and locking of the operative frequency of the delay line (e.g. minimizing the power at the cross output port).

\section{A. Characterization of the Tunable Coupler}

The transfer function of the designed tunable coupler based on a balanced Mach-Zehnder interferometer was characterized on an isolated test device identical to that integrated in the delay lines. Figure 6(a) reports the normalized power transmission of the bar [blue dots] and cross ports [orange dots] of the coupler as function of the current $\mathrm{I}_{\mathrm{c}}$ fed into the phase

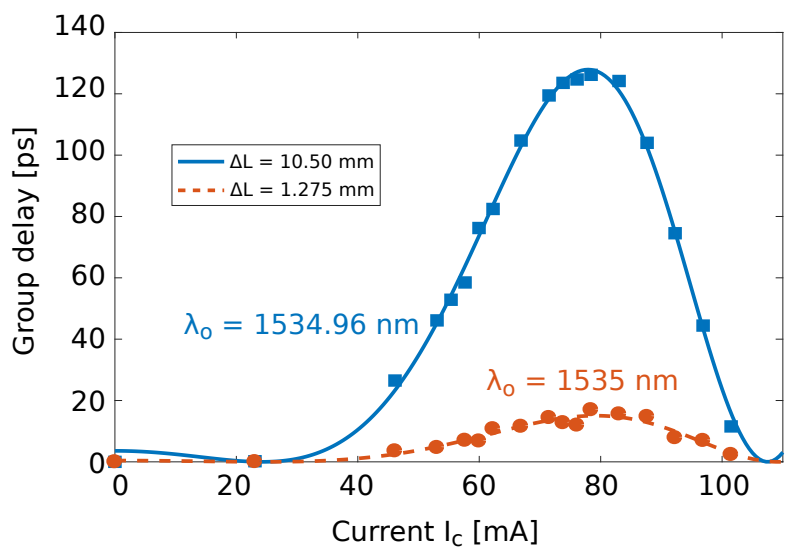

Fig. 7. Measured group delay as function of the current $I_{C}$ fed to the tunable couplers for the delay line with $\Delta \mathrm{L}=10.5 \mathrm{~mm}$ [blue squares] and $\Delta \mathrm{L}=$ $1.275 \mathrm{~mm}$ [orange circles]. Blue solid line and orange dashed line represent the fit with a squared cosine function in the two cases.

shifter at $\lambda=1550 \mathrm{~nm}$ for TE polarized input light. The same results were obtained for the TM mode. Blue solid and orange dashed lines represent the fit performed on the experimental data at bar and cross ports with a squared sine and squared cosine functions, respectively. The normalized power at the cross port represents the power coupling factor K used in Sec. II. Despite this device is expected to couple all the power to the cross port when the phase shifter is not used, the realized device is almost on the opposite condition, with $85 \%$ of the power on the bar port for $\mathrm{I}_{\mathrm{c}}=0 \mathrm{~mA}$. For $\mathrm{I}_{\mathrm{c}}=34 \mathrm{~mA}$ power is completely coupled at the bar output port $(\mathrm{K}=0)$, with $\mathrm{I}_{\mathrm{C}}=$ $60 \mathrm{~mA}$ the $3-\mathrm{dB}$ condition is reached $(\mathrm{K}=0.5)$ while at about $\mathrm{I}_{\mathrm{C}}=75 \mathrm{~mA}$ input power is coupled to the cross output port ( $\mathrm{K}$ $=1$ ). This behaviour is well repeatable over different identical tunable couplers realized on the same fabrication run along with the tunable delay lines. Figure 6(b) shows the measured power transmission spectra at the cross port for $\mathrm{I}_{\mathrm{C}}=57 \mathrm{~mA}$ (close to $3-\mathrm{dB}$ condition, dotted line) and $\mathrm{I}_{\mathrm{c}}=73 \mathrm{~mA}$ [solid line] for TE mode. As can be seen the behaviour of the coupler can be considered constant over a bandwidth of at least 60 $\mathrm{nm}$, much larger than the operative bandwidth of the designed delay lines, with only a smooth wavelength dependence that is almost invariant for different fed currents. The same results were obtained at the bar port and for different current values.

\section{B. Tunable Delay Line}

In order to characterize the functionality of the delay lines, input and output ports were selected as represented schematically in Fig. 1. The responses of the two realized devices were measured by means of an Optical Vector Analyser that allowed to characterize both the power transmission spectrum and the phase spectrum, that was then used to retrieve the group delay spectrum. During the measurements, the same current $I_{c}$ was fed to the two tunable couplers of the delay lines to obtain the same $\mathrm{K}$ value. The shift of the operative frequency due to the thermal tuning of the couplers (thermal cross-talk) was compensated through the phase shifter integrated on the short branch of the Mach-Zehnder. 

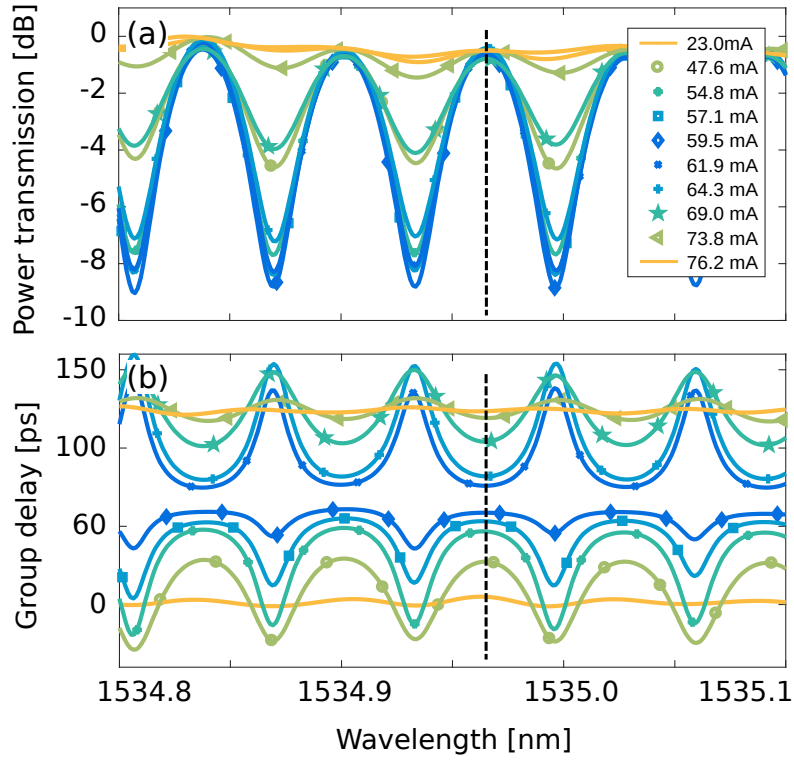

Fig. 8. (a) Power transmission spectrum and (b) group delay spectrum for the Mach-Zehnder delay line with an unbalance $\Delta \mathrm{L}=10.5 \mathrm{~mm}$

The realized long delay line has an unbalance between the two arms of the Mach-Zehnder of $10.5 \mathrm{~mm}$, corresponding to an expected group delay variation for the TE polarized mode of about $123 \mathrm{ps}$. The measured group delay as function of the current $I_{c}$ fed into the couplers at the operative wavelength $\lambda_{o}=1534.96 \mathrm{~nm}$ for TE polarized input light is shown in Fig. 7 with blue square marks, normalized to the minimum measured delay. Solid blue line represents the fit performed on the experimental data with a squared cosine model. As can be seen, results reported in Fig. 7 are in very good agreement with the characterization of the isolated tunable coupler [Fig. 6, dashed orange line], confirming the linear relationship $\tau\left(f_{o}\right)=$ $\tau_{0}+T K$ between the group delay and the coupling coefficient. The minimum delay is achieved with a $\mathrm{I}_{\mathrm{c}} \simeq 24 \mathrm{~mA}(\mathrm{~K}=0)$, causing light to travel in the shortest branch of the MachZehnder interferometer. On the contrary, light travels only in the longest branch of the delay line (maximum delay) for $I_{C}$ $\simeq 78 \mathrm{~mA}(\mathrm{~K}=1)$. The measured variation of the group delay at the operative wavelength is about $124 \mathrm{ps}$, corresponding to a FSR of $8.1 \mathrm{GHz}$, in accordance with the designed value.

The normalized power transmission and group delay spectra measured for the device between $\lambda=1534.8 \mathrm{~nm}$ and $\lambda$ $=1535.1 \mathrm{~nm}$ are shown in Fig. 8 (a) and (b) for some current values (TE mode). The dashed black lines mark the operative wavelength $\lambda_{o}=1534.96 \mathrm{~nm}$. The measured spectral behaviour is in agreement with the simulations shown in Fig. 2. For $\mathrm{I}_{\mathrm{c}}=23 \mathrm{~mA}(\mathrm{~K} \simeq 0)$ power transmission and group delay are almost wavelength independent and the latter is at minimum [yellow lines]. In this condition (light travelling in the shortest branch of the delay line), the estimated overall insertion loss of the device is about $5 \mathrm{~dB}$, excluding fibre coupling losses. Increasing the applied current (and hence $\mathrm{K}$ ), maxima and minima appear in the spectra [green to blue marks]. At the operative wavelength normalized power

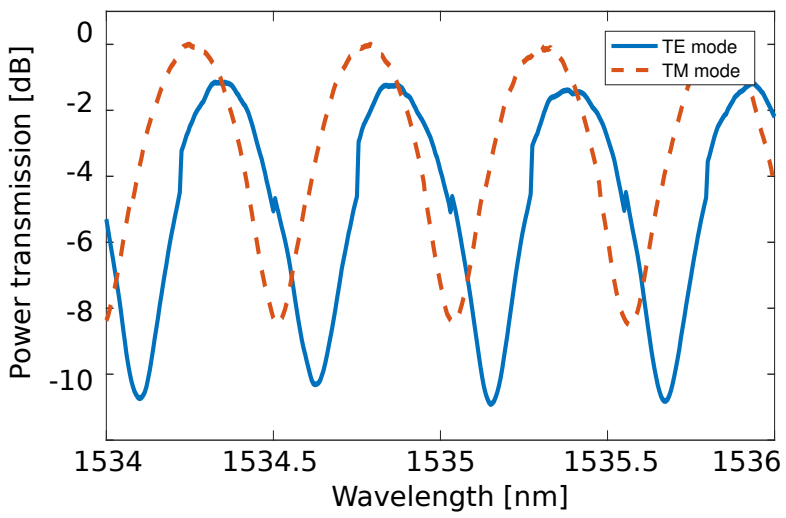

Fig. 9. Power transmission spectra of the delay line with $\Delta \mathrm{L}=1.275 \mathrm{~mm}$ for TE (blue solid line) and TM (orange dashed line) polarized input light.

transmission is almost constant while group delay steadily increases. For $\mathrm{I}_{\mathrm{c}}=59.5 \mathrm{~mA}(\mathrm{~K} \simeq 0.5)$ the deepest notches appear in the power spectrum while the group delay is almost spectrally flat at a level that is half of the maximum tuning allowed by the device $(62 \mathrm{ps})$. With $\mathrm{I}_{\mathrm{c}}=76.2 \mathrm{~mA}$ (K $\simeq 1$ ), both power transmission and group delay are again wavelength independent and the latter reaches the maximum of 124 ps [yellow line]. At $\mathrm{I}_{\mathrm{c}}=59.5 \mathrm{~mA}$, the $3-\mathrm{dB}$ bandwidth measured on the power transmission spectrum around the marked operative wavelength is $4.45 \mathrm{GHz}$.

Similar results were obtained also with the short delay line, as reported in Fig. 7 with orange circles for TE polarized mode. Results of the fit with a squared cosine model are reported with a dashed orange line. In this case the unbalance of the Mach-Zehnder is $\Delta \mathrm{L}=1.275 \mathrm{~mm}$, with a designed tunability of the group delay of 15 ps. The behaviour of the delay line is the same of the previous case, with an operative wavelength $\lambda_{o}=1535 \mathrm{~nm}$. The maximum measured delay variation of $14.9 \mathrm{ps}$ is reached at $\mathrm{I}_{\mathrm{c}} \simeq 79 \mathrm{~mA}(\mathrm{~K} \simeq 1)$ and corresponds to a FSR of $67.06 \mathrm{GHz}$. The spectral behaviour is very similar to that reported for the first delay line and in well agreement with the simulations. At $\mathrm{I}_{\mathrm{c}}=59.5 \mathrm{~mA}(\mathrm{~K}$ $\simeq 0.5$ ), the $3-\mathrm{dB}$ bandwidth around the operative frequency is about $35 \mathrm{GHz}$ (again measured on the power transmission spectrum). As expected, this device can be used to control the delay of signals with a much wider bandwidth compared to the 10.5-mm-unbalanced device, at the expense of a smaller tunability range.

\section{Polarization Dependence}

The delay line with $\Delta \mathrm{L}=1.275 \mathrm{~mm}$ was exploited to characterize the dependence of the device to the polarization of the input light. Polarization conversion is not expected to be a particular issue for the exploited waveguide and bending radius [Fig. 5] and it is hence neglected [8]. Figure 9 reports the measurement of the power transmission spectra for the $\mathrm{TE}$ and $\mathrm{TM}$ polarized input modes with $\mathrm{I}_{\mathrm{c}}=59.5 \mathrm{~mA}(\mathrm{~K}$ $\simeq 0.5$ ). Measurements were performed with exactly the same condition of the experimental setup, including fibre alignment. Polarization dependent loss (measured on the maxima of the 

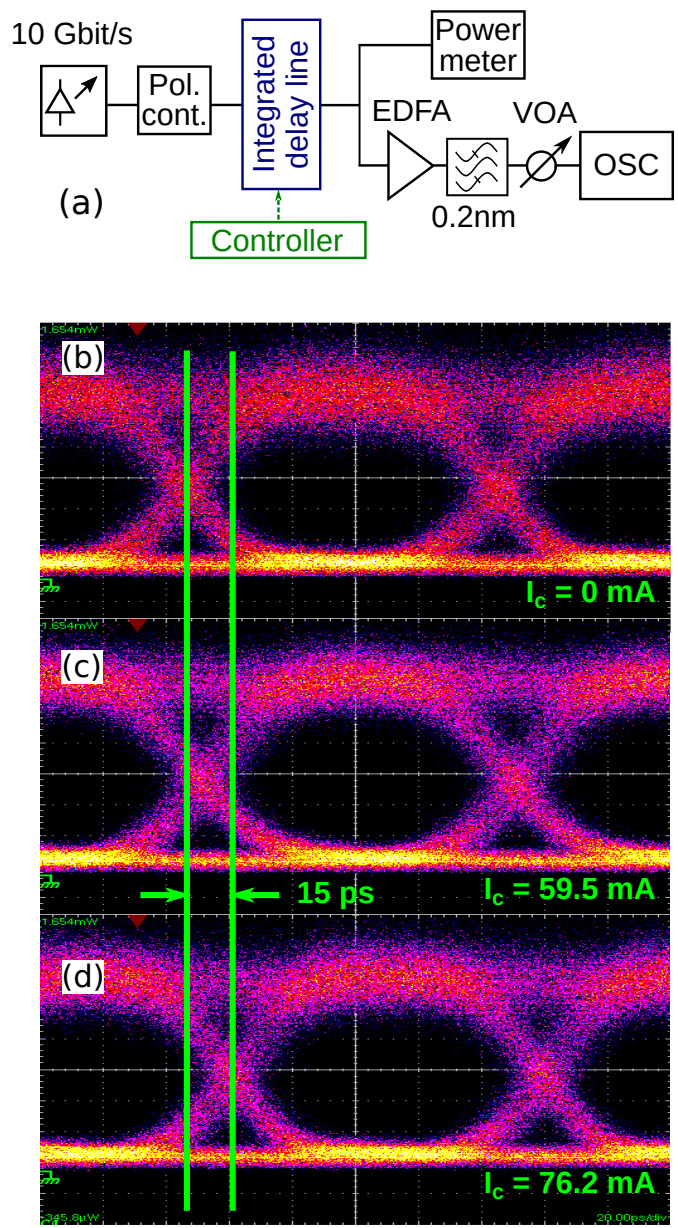

Fig. 10. Propagation delay control of a $10 \mathrm{Gbit} / \mathrm{s}$ OOK NRZ signal through the proposed delay line with $\Delta \mathrm{L}=1.275$. (a) Experimental setup. (b-d) Measured eye diagrams when the current fed to the two tunable couplers is $0 \mathrm{~mA}$ (minimum delay), $59.5 \mathrm{~mA}$ (delay increased of about $7.4 \mathrm{ps}$ ) and $76.2 \mathrm{~mA}$ (maximum delay increase, $15 \mathrm{ps}$ ).

two spectra) is in the order of $1.4 \mathrm{~dB}$. This value was measured also on different devices on the same samples and is mainly due to a slight difference on the coupling efficiency at chip facets. The wavelength shift between the two spectra is 1.12 $\mathrm{nm}$, corresponding to a birefringence of the effective indices of about $2.6 \cdot 10^{-3}$, that is in good agreement with the simulation results for the considered waveguide. Lastly, TE and TM FSR are $67.06 \mathrm{GHz}$ and $67.13 \mathrm{GHz}$, respectively. The measured group index birefringence is hence about $4 \cdot 10^{-3}$ (again in good agreement with simulations), leading to a negligible differential group delay of about $20 \mathrm{fs}$, that is $0.1 \%$ of the maximum obtainable delay variation. With the same group index birefringence, the long delay line with $\Delta \mathrm{L}=10.5 \mathrm{~mm}$ exhibits again a negligible differential group delay between the two polarization states of about 0.14 ps.

\section{Continuous Control of the Delay with $10 \mathrm{Gbit} / \mathrm{s}$ Signals}

The proposed optical delay line was finally tested within the transmission system sketched in Fig. 10(a). A $10 \mathrm{Gbit} / \mathrm{s}$ OOK NRZ channel was generated at a wavelength around $1535 \mathrm{~nm}$ and after polarization adjustment was coupled to the short delay line. The minimum bandwidth of the device, larger than $30 \mathrm{GHz}$ [Sec. III-B], is sufficiently wide to avoid any distortion to the transmitted impulses. At the output, the channel was collected and sent into an optical oscilloscope after amplification and filtering. Through the controller, several working points were selected for the delay line, with $\mathrm{I}_{\mathrm{c}}$ comprised between $0 \mathrm{~mA}$ and $76.2 \mathrm{~mA}$. The operative wavelength was tuned by means of the phase controller integrated in the MachZehnder. Figures 10(b-d) show the eye diagrams measured at the oscilloscope for different currents fed into the tunable couplers. As can be seen, a good eye-opening is preserved in all the cases without any degradation related to a tuning of the control current. Consistently with the characterizations shown in Sec. III-B, the delay of the transmitted signal can be continuously controlled up to a maximum of about 15 ps without distortions, confirming the functionality of the proposed delay line.

\section{Conclusion}

In conclusion, we have proposed and experimentally demonstrated an integrated device implementing a non-resonant optical delay line. Exploiting a Mach-Zehnder interferometer equipped with two tunable couplers, this solution allows to obtain true-time delay operation on a bandwidth consistently larger than ring-based delay lines, reducing the induced dispersion and limiting the effect of propagation losses. The group delay is continuously tuned by varying the coupling ratio of the two couplers of the same quantity, preventing amplitude modulations due to the reconfiguration of the delay line. The experimental characterization of the realized device proved a group delay tunability up to $125 \mathrm{ps}$ and the functionality was exploited also to control the delay of a $10 \mathrm{Gbit} / \mathrm{s}$ data stream. Larger delays could be easily obtained properly designing the unbalance of the Mach-Zehnder to obtain a trade-off between delay and operational bandwidth.

\section{REFERENCES}

[1] A. Melloni, F. Morichetti, C. Ferrari, and M. Martinelli, "Continuously tunable 1 byte delay in coupled-resonator optical waveguides," Optics letters, vol. 33, no. 20, pp. 2389-2391, 2008.

[2] F. Xia, L. Sekaric, and Y. Vlasov, "Ultracompact optical buffers on a silicon chip," Nature photonics, vol. 1, no. 1, pp. 65-71, 2007.

[3] H. Park, J. P. Mack, D. J. Blumenthal, and J. E. Bowers, "An integrated recirculating optical buffer," Optics express, vol. 16, no. 15, pp. 11124 $11131,2008$.

[4] J. Capmany and D. Novak, "Microwave photonics combines two worlds," Nature photonics, vol. 1, no. 6, pp. 319-330, 2007.

[5] E. Choi, J. Na, S. Y. Ryu, G. Mudhana, and B. H. Lee, "All-fiber variable optical delay line for applications in optical coherence tomography: feasibility study for a novel delay line," Optics express, vol. 13, no. 4, pp. 1334-1345, 2005

[6] I. Frigyes and A. Seeds, "Optically generated true-time delay in phasedarray antennas," IEEE Transactions on Microwave Theory and Techniques, vol. 43, no. 9, pp. 2378-2386, 1995.

[7] A. Meijerink, C. G. Roeloffzen, R. Meijerink, L. Zhuang, D. A. Marpaung, M. J. Bentum, M. Burla, J. Verpoorte, P. Jorna, A. Hulzinga et al., "Novel ring resonator-based integrated photonic beamformer for broadband phased array receive antennas-Part I: Design and performance analysis," Journal of Lightwave Technology, vol. 28, no. 1, pp. 3-18, 2010. 
[8] M. Smit, X. Leijtens, H. Ambrosius, E. Bente, J. van der Tol, B. Smalbrugge, T. de Vries, E.-J. Geluk, J. Bolk, R. van Veldhoven, L. Augustin, P. Thijs, D. DAgostino, H. Rabbani, K. Lawniczuk, S. Stopinski, S. Tahvili, A. Corradi, E. Kleijn, D. Dzibrou, M. Felicetti, E. Bitincka, V. Moskalenko, J. Zhao, R. Santos, G. Gilardi, W. Yao, K. Williams, P. Stabile, P. Kuindersma, J. Pello, S. Bhat, Y. Jiao, D. Heiss, G. Roelkens, M. Wale, P. Firth, F. Soares, N. Grote, M. Schell, H. Debregeas, M. Achouche, J.-L. Gentner, A. Bakker, T. Korthorst, D. Gallagher, A. Dabbs, A. Melloni, F. Morichetti, D. Melati, A. Wonfor, R. Penty, R. Broeke, B. Musk, and D. Robbins, "An introduction to inp-based generic integration technology," Semiconductor Science and Technology, vol. 29, no. 8, p. 083001, 2014.

[9] N. Tessema, Z. Cao, J. Van Zantvoort, K. Mekonnen, A. Dubok, E. Tangdiongga, A. Smolders, and A. Koonen, "A Tunable $\mathrm{Si}_{3} \mathrm{~N}_{4}$ Integrated True Time Delay Circuit for Optically-Controlled K-Band Radio Beamformer in Satellite Communication," Journal of Lightwave Technology, vol. 34, no. 20, pp. 4736-4743, 2016.

[10] G. Lenz, B. Eggleton, C. K. Madsen, and R. Slusher, "Optical delay lines based on optical filters," IEEE Journal of Quantum Electronics, vol. 37, no. 4, pp. 525-532, 2001.

[11] R. Bonjour, S. A. Gebrewold, D. Hillerkuss, C. Hafner, and J. Leuthold, "Continuously tunable true-time delays with ultra-low settling time," Optics express, vol. 23, no. 5, pp. 6952-6964, 2015.

[12] J. Cardenas, M. A. Foster, N. Sherwood-Droz, C. B. Poitras, H. L. Lira, B. Zhang, A. L. Gaeta, J. B. Khurgin, P. Morton, and M. Lipson, "Wide-bandwidth continuously tunable optical delay line using silicon microring resonators," Optics express, vol. 18, no. 25, pp. 26 525-26 534, 2010.

[13] A. Canciamilla, M. Torregiani, C. Ferrari, F. Morichetti, R. De La Rue, A. Samarelli, M. Sorel, and A. Melloni, "Silicon coupled-ring resonator structures for slow light applications: potential, impairments and ultimate limits," Journal of Optics, vol. 12, no. 10, p. 104008, 2010.

[14] A. Melloni, A. Canciamilla, C. Ferrari, F. Morichetti, L. O'Faolain, T. Krauss, R. De La Rue, A. Samarelli, and M. Sorel, "Tunable delay lines in silicon photonics: coupled resonators and photonic crystals, a comparison," IEEE Photonics Journal, vol. 2, no. 2, pp. 181-194, 2010

[15] F. Morichetti, A. Melloni, A. Breda, A. Canciamilla, C. Ferrari, and M. Martinelli, "A reconfigurable architecture for continuously variable optical slow-wave delay lines," Optics Express, vol. 15, no. 25, pp. 17273-17282, 2007.

[16] J. B. Khurgin and P. A. Morton, "Tunable wideband optical delay line based on balanced coupled resonator structures," Optics letters, vol. 34 no. 17 , pp. 2655-2657, 2009.

[17] R. L. Moreira, J. Garcia, W. Li, J. Bauters, J. S. Barton, M. J. Heck, J. E. Bowers, and D. J. Blumenthal, "Integrated ultra-low-loss 4-bit tunable delay for broadband phased array antenna applications," IEEE Photon. Technol. Lett, vol. 25, no. 12, pp. 1165-1168, 2013.

[18] M. S. Rasras, C. K. Madsen, M. A. Cappuzzo, E. Chen, L. T. Gomez, E. J. Laskowski, A. Griffin, A. Wong-Foy, A. Gasparyan, A. Kasper et al., "Integrated resonance-enhanced variable optical delay lines," IEEE photonics technology letters, vol. 17, no. 4, pp. 834-836, 2005.

[19] M. V. Drummond, R. N. Nogueira, P. P. Monteiro, M. A. Violas, C. Sterner, and P. Y. Fonjallaz, "Tunable optical dispersion compensator based on power splitting between two dispersive media," Journal of Lightwave Technology, vol. 28, no. 8, pp. 1164-1175, April 2010.

[20] M. V. Drummond, P. P. Monteiro, and R. N. Nogueira, "Photonic truetime delay beamforming based on polarization-domain interferometers," J. Lightwave Technol., vol. 28, no. 17, pp. 2492-2498, Sep 2010.

[21] V. C. Duarte, M. V. Drummond, and R. N. Nogueira, "Photonic truetime-delay beamformer for a phased array antenna receiver based on self-heterodyne detection," J. Lightwave Technol., vol. 34, no. 23, pp. 5566-5575, Dec 2016.

[22] Y. Liu, A. Wichman, B. Isaac, J. Kalkavage, E. Adlest, T. Clark, and J. Klamkin, "Single ring resonator delays for integrated optical beam forming networks," in 2016 IEEE International Topical Meeting on Microwave Photonics (MWP), Oct 2016, pp. 321-324.

[23] JePPIX, http://www.jeppix.eu.

[24] D. Melati, F. Morichetti, A. Canciamilla, D. Roncelli, F. Soares, A. Bakker, and A. Melloni, "Validation of the building-block-based approach for the design of photonic integrated circuits," Journal of Lightwave Technology, vol. 30, no. 23, pp. 3610-3616, 2012.

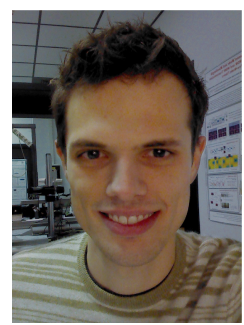

Daniele Melati is a postdoctoral researcher at $\mathrm{Po}$ litecnico di Milano. He received the M.Sc. degree in Telecommunication Engineering and the Ph.D. degree (graduated with honour and European Ph.D. title) in Information Engineering from Politecnico di Milano. He temporarily joined the Photonic Integration Group at the Eindhoven University of Technology, The Netherlands, as visiting researcher. His main research interests include photonic device modelling with a focus on Process Design Kit development, stochastic analysis and design optimization; novel test-on-wafer approaches; effects of thermal fluctuations and crosstalk on photonic circuits; design and development of photonic circuits for mode-division multiplexing systems; advanced experimental techniques. $\mathrm{He}$ actively collaborates with the company Filarete to the development of the circuit simulator for optical circuits ASPIC. Since 2009 he was involved in several European collaborative projects.



Abi Waqas is a $\mathrm{PhD}$ student at Politecnico di Milano with Photonic Devices group. He received the B.E degree in Telecommunication Engineering in 2012 and completed his M.E in Telecommunication Engineering and Management in 2014 from Mehran University of Engineering and Technology, Pakistan. His research interests are modelling, characterization and statistical analysis of photonic integrated devices and performance analysis of free space optics systems. He was an exchange student for 1 year in the Aalborg University of Copenhagen, Denmark in 2010. He worked as a Research associate in MUET Pakistan for 6 months and has also served in Eni Pakistan Limited as a Trainee Network Engineer for two years. He is currently (on leave) a Lecturer in the department of Telecommunication Engineering, Mehran University, Pakistan.

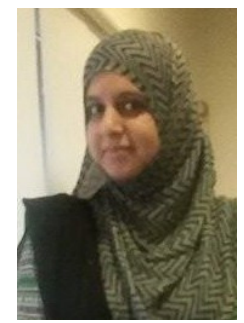

Zarlish Mushtaq received the B.E degree in Telecommunication Engineering in 2012 and completed her M.E in Telecommunication Engineering and Management in 2014 from, Mehran University of Engineering and Technology, Pakistan. She served in Augere Limited for the period of 1 year. She started her PhD in Photonics in Mehran University of Engineering and Technology, Pakistan in 2016. She is awarded Erasmus Mundus INTACT Exchange Scholarship to pursue $\mathrm{PhD}$ with Photonics Devices Group in Politecnico Di Milano, Italy.

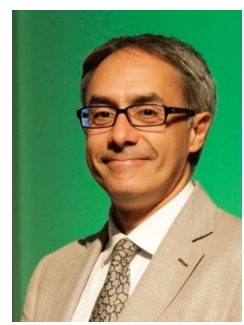

Andrea Melloni is Full Professor at Dipartimento di Elettronica, Informazione e Bioingegneria Politecnico di Milano where he leads the group of Photonic Devices and teaches the master course "Photonics Devices". With a background in microwaves, his field of research is in the analysis, design, characterization and exploitation of passive integrated optical devices for telecom and sensing. He has been one of the pioneers of the slow light concept and its exploitation in the linear and nonlinear domain. $\mathrm{He}$ is currently contributing to define the new schemes of generic photonic foundries in Europe. In 2008 he founded the company Filarete, for the development and commercialization of the first circuit simulator for integrated optical circuits, ASPIC (www.aspicdesign.com). He holds 13 international patents in the field of integrated optics and components and he is author and co-author of over 90 publications on the major international journals. 